\title{
Recreational Problem Solving Activi- ties in the Italian Language Classroom
}

\section{Marcel Danesi}

\section{Introduction}

In recent years, there has been an ever-increasing tendency to provide "recreational" activities in foreign language manuals. ${ }^{1}$ This bears witness to a trend - traceable probably to the upsurge in cognitive psychology in education - that problem solving activities are not only enjoyable in themselves but are also conducive to long-term memory storage and therefore to successful learning. The abundant psychological literature on problem solving points, in general, to its effectiveness as a teaching/ learning strategy. ${ }^{2}$ Teachers of mathematics in particular have always maintained that problem solving is a crucial pedagogical technique in teaching mathematical principles and concepts. These teachers have discovered, additionally, that most students find problem solving a rewarding and stimulating activity. As the great English puzzlist, Henry E. Dudeney, once wrote, a "good puzzle, like virtue, is its own reward. Man loves to be confronted by a mystery, and he is not entirely happy until he has solved it."

More and more, teachers of foreign languages are starting to realize that they too can make use of problem solving activities as heuristic teaching devices. Anthony Mollica, who has become a leading advocate of the incorporation of puzzles and games into the pedagogical routine, suggests in a recent key article that such activities "will not only inject a recreational element into the learning process, but will also foster verbal learning itself and stimulate communicative interaction. ${ }^{\prime 4}$

On the basis of personal teaching experience and that of certain colleagues, and on the basis of the literature on this subject, ${ }^{5}$ the purpose of this study is to suggest and describe various recreational problem solving activities for Italian language courses. Specifically, I will deal with the why, what and how of puzzles and games in the teaching of Italian as a second, or foreign, language. 
Why Recreational Problem Solving Activities in Language Teaching?

It is first of all necessary to clarify what is meant by "recreational" problem solving activities. These are activities which require the cognitive mechanisms involved in any problem solving act but which are recreational in intent. Belonging to this category are such well known puzzles and games as crosswords, scrambled letters, "logic" puzzles, etc. The question that comes immediately to mind is as follows: What purpose do these activities serve in foreign language courses? In brief, the literature on this subject suggests that there are solid psychological and pedagogical reasons for the use of such activities in language teaching. In the case of Italian, moreover, one can add a cultural purpose as well because Italians have always enjoyed crosswords, anagrams, etc. as a form of recreation.

From a psychological standpoint, it is frequently pointed out that problem solving and language learning are interrelated. Clark, for example, suggests that the reasoning used in problem solving "is accomplished mainly through certain very general linguistic processes, the same mental operations that are used regularly in understanding language. ${ }^{\prime 6}$ What this implies is that problem solving activities are supportive of language learning because they require the knowledge and use of a language's structural and lexical forms in arriving at a solution - the goal of any problem solving activity. As a consequence, these activities force the student to "think" in the target language because they involve cognitive processes closely linked to both the receptive and productive dimensions of language learning. In other words, students must first decipher the cues required to understand and solve the problem in the target language and subsequently formulate their solution in terms of the target language. To quote Scribner, problem solving, "involves relating or integrating the information presented in the individual sentences and assimilating it to existing lexical and nonlexical knowledge schemas. ${ }^{77}$

In addition to psychological reasons, it is often pointed out that problem solving activities have various pedagogical functions. Above all else, they are motivational stimuli. In the words of Gardner, these activities relieve the tedium of pedagogical routine "by injecting recreational topics in a course, topics strongly tinged with elements of play, humour, beauty, surprise." 8 Moreover, they can be used, like more traditional exercises, to practice or review some point of grammar or vocabulary. The difference between traditional exercises and recreational problem solving activ- 
ities lies primarily in the fact that in the latter there is no overt structural or lexical control, whereas in traditional drills and exercises (substitutions, transformations, etc.) the purpose is to practice some pre-determined language goal (e.g., noun plural formation). In recreational problem solving activities, language-based goals are not always evident. Nevertheless, as will be discussed shortly, these activities may be utilized in such a way so as to serve various language-based purposes.

Finally, it can be said that in the case of Italian, problem solving activities of a recreational nature also fulfill a cultural purpose. They exemplify a type of activity that Italians like to do often, as evidenced by the large number of "riviste enigmistiche" published in Italy and always visible in bookstores and on newsstands. In fact, it can be argued with some reason that "l'enigmistica" constitutes one of the most popular "passatempi" of Italians. Thus, the use of recreational problem solving activities in Italian language courses not only fulfills psychological and pedagogical needs, but is also indicative of an important component of Italian culture.

\section{What Types of Recreational Problem Solving Activities?}

The first obvious practical problem connected with the use of recreational problem solving activities is to decide which kinds of activities are appropriate for some learning goal. Clearly, those kinds of activities which require some special skill or technical know-how are of no pedagogical value. On the other hand, those which are familiar and which require only direct thinking are possible candidates for any typology of recreational problem solving activities. Basically, two main types can be signalled out as possible genres in terms of their solution focus. The type which focuses on the language itself (or any of its parts) may be called form-based; the type that focuses instead on some non-linguistic solution may be designated content-based. In other words, if the attention of the problem solver is directed to some structural or lexical aspect of the language (e.g., crosswords), then the puzzle is of the formbased variety. If, on the other hand, the problem solver's attention is directed to a non-linguistic solution (e.g., a problem in recreational logic), then the puzzle is of the content-based type.

Mollica has correctly pointed out that, in addition to the solution focus, the medium in which a puzzle is formulated is an important classificatory criterion. ${ }^{9}$ According to Mollica, puzzles can be classified as either "printed" or "visual." Printed puzzles are 
based on the printed word, whereas visual ones are formulated in terms of pictures or drawings. Although I will use printed puzzles to exemplify both form-based and content-based types, what I have to say is also applicable to the visual puzzle variety.

\section{Form-Based Puzzles}

Form-based puzzles have become quite popular in foreign language teaching for obvious reasons. Puzzles such as crosswords or scrambled letters are effective pedagogical devices for the practice and/or review of lexical material, as well as for the recognition of word-formation patterns.

Perhaps the most popular form-based puzzle is the crossword. This is a type familiar to almost everyone and need not be described here. Essentially, crosswords are useful for the practice and/or review of lexical material. ${ }^{10}$

Also very popular are the so-called scrambled letter and scrambled word puzzle types. The former requires the problem solver to unscramble letters to form words (e.g., $r, e, a, g, m, n, a, i \downarrow$ mangiare); the latter instead requires the problem solver to form sentences from scrambled words (e.g., il, italiano, ragazzo, parla $\emptyset$ Il ragazzo parla italiano). Such puzzles are extremely useful for teaching word and sentence structure respectively.

Recently, the "crucipuzzle" or word search puzzle has become a popular pedagogical activity. This puzzle type requires the problem solver to find words hidden in a word maze. The following simple example contains the hidden words amico, Marco and ora:

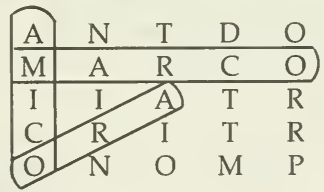

Word searches are obviously useful in teaching students to recognize word patterns and for lexical practice and/or review.

To this list of well known form-based puzzles, I would like to add cryptograms, alphametics, palindromes and deletions. Cryptograms are puzzles in code writing. They require the problem solver to substitute letters for other letters or numbers in such a way that a message can be decoded. For example, the solution to the following cryptogram is: $1=l, 2=t, 3=u, 4=r, 5=g, 6=z, 7=e$, $8=a$ : 


\section{$\begin{array}{lllll}18 & 4858668 & 17557 & 18 & 1722348\end{array}$ \\ La ragazza legge la lettura}

This puzzle type is particularly effective for the teaching of the recognition of word formation patterns and of predictable morphosyntactic items (articles, suffixes, etc.).

Alphametics (also known as cryptarithms) are puzzles which require the problem solver to replace letters with digits in order to reconstruct the original numerical layout. Each alphametic is a problem in simple arithmetic. ${ }^{11}$ For example, one possible solution to the following problem is: $l=7, a=0, u=5, m=2, s,=9, f$ $=1$ :

$\begin{array}{r}\text { LA } \\ + \text { S UA } \\ \hline \text { F A M A } \begin{array}{r}70 \\ 10\end{array}++950 \\ \hline 1020\end{array}$

The idea in such puzzles is that the letters must form meaningful words or phrases. These interesting puzzles can be used as mnemonic devices to reinforce certain lexical or grammatical points. In the puzzle above, the grammatical point being illustrated is, of course, the possessive adjective.

Palindromes require the problem solver to find words that can be read both from left to right and from right to left (e.g., oro, esse, osso, etc.). This puzzle type focuses on morphemic structure and is, of course, useful for lexical reinforcement.

Deletions are puzzles consisting of sentences in which parts of words have been deleted. The idea is to reconstruct the entire sentence:

$$
\begin{array}{lllll}
\text { Que... uom... ha..o gi. } m . \text { ngiat. } & \text { ha } \\
\text { Quegli } & \text { uomini hanno già mangiato }
\end{array}
$$

This type of puzzle is useful for teaching lexical and structural features, and also for teaching discourse expectancy.

Various other types of form-based puzzles can be added to the list of recreational problem solving activities. ${ }^{12}$ The idea behind these puzzles is that the problem solver's attention is directed to the form of the language itself. They are, therefore, similar to traditional exercises and drills. But since they are recreational in na- 
ture they add an important fun element to the learning process. Teachers can easily construct form-based puzzles by themselves or obtain them from the many puzzle magazines published in Italy. ${ }^{13}$

\section{Content-Based Puzzles}

Unlike the puzzles described above, content-based puzzles direct the problem solver's attention to their content. These puzzles are, in my opinion, particularly useful for the cultivation of global linguistic competence because they require an interpretation of the language in which they are formulated in order to arrive at a solution. Three types of content-based puzzles are, in my view, especially useful: word tricks, logical deductions and verbal equations. ${ }^{14}$

Word tricks, as their name implies, are deceptive statements which require the problem solver to scrutinize the language of the puzzle very closely:

Il treno che va da Roma a Bologna viaggia alla velocità di 150 chilometri all'ora. Il treno che va da Bologna a Roma parte un'ora dopo e viaggia alla velocità di solo 100 chilometri all'ora. Quando i due treni si incontrano, quale treno sarà piú vicino a Roma?

The solution to this problem is obvious only when one considers what it means when the two trains meet each other. Clearly, when the two trains meet each other they do so at a single point, and therefore neither one is nearer to Rome. Problems of this type are tricky because they simulate the style of problems of arithmetic or algebra. The problem solver must penetrate this distracting stylistic façade in order to arrive at the proper solution. This puzzle type is therefore quite useful for cultivating global linguistic competence because it requires the problem solver to decipher and assess the content correctly, and then to formulate an answer in the target language.

Unlike word tricks, logical deductions do not involve any deliberately deceptive statements. These puzzles simply require logical thinking for their solution:

Gianni, Carlo, Maria e Lucia esercitano le seguenti professioni, ma non necessariamente rispettivamente: artista, dentista, pianista, e farmacista. Il farmacista, che ha una sorella artista, è sposato. Maria non se ne intende affatto di arte e non suona nessuno strumento musicale. Gianni non è sposato. Chi è il dentista? 
Since the pharmacist is a married man and since Gianni is not married, it can be deduced that Carlo is the pharmacist. Since Carlo's sister is an artist and since Maria knows nothing about art, it can be further deduced that Lucia is the artist. Moreover, since Maria does not play a musical instrument, the pianist must therefore be Gianni. Consequently, Maria is the dentist. There are many types of logical deductions; but it is beyond the scope of this paper to discuss them in detail. ${ }^{15}$ Suffice it to say that they all involve the same solution strategy; namely, the setting up of hypotheses and determining their consistency in terms of the total framework of the problem. Like word tricks, these puzzles require the problem solver to decipher and assess their content, and then to activate a solution strategy in the target language.

Verbal equations are the simple "story" problems of arithmetic and algebra:

Marco e Dino hanno, insieme, 40 anni. Marco è più vecchio di Dino. La differenza fra le loro età è uguale a 10 anni. Quanti anni ha Marco?

By letting Marco's age be represented by $x$ and Dino's age by 40 $x$, it can be readily seen that $x-(40-x)=10$. Solving this equation, one finds that $x=25$, which is, of course, Marco's age. Like the previous two content-based puzzle types, verbal equations require the problem solver to interpret the language of the puzzle correctly and then to activate a solution strategy.

Various other content-based puzzle types can be added to the list of recreational problem solving activities for language courses. Like form-based puzzles, teachers can make these up by themselves, or adapt them from magazines or puzzle anthologies. ${ }^{16}$ Since they direct the problem solver's attention to the target language content, they can be used as instruments for the cultivation of overall, or global, linguistic competence.

How Can Recreational Problem Solving Activities Be Used as Learning/ Teaching Devices?

Perhaps the most important question connected with the use of recreational problem solving activities in language courses is to decide if they are appropriate for a particular group of students. The use of such activities is, needless to say, a matter of preference. Although the popularity of puzzles such as the ones described above is attested by the fact that few daily newspapers do not contain a puzzle corner or department, not everyone will 
enjoy solving puzzles. If, however, the decision is made to adapt recreational problem solving activities to language teaching, then there are at least two major pedagogical questions that face the teacher with regard to how they can be utilized and integrated in the language program: (1) the compilation of suitable puzzles; (2) the instructional "mechanics" involved.

The compilation of puzzles is a relatively simple task. Most, if not all, of the puzzles described in this paper can be easily constructed by teachers themselves. This allows teachers to control beforehand the structural and lexical content of the puzzles and therefore to make them immediately suitable for predetermined pedagogical purposes. In other words, they can be "tailored" to meet the teaching/learning task at hand. The other alternative is, of course, to select puzzles from published sources. As already mentioned, there are many "riviste enigmistiche" published in Italy which contain a wide variety of puzzle types. In addition, there are many puzzle anthologies published in English from which the teacher can select appropriate puzzles and translate them into Italian. Puzzles selected in this way will probably have to be "edited" in some way to reflect both the level of competence of the students and the teaching/learning goals they are intended to serve.

The second pedagogical question with regard to recreational problem solving activities concerns the instructional "mechanics" related to their use in language courses. Form-based puzzles are clearly useful for grammatical and lexical practice and/or instruction. Teachers may wish to present some point in grammar or vocabulary with a form-based puzzle in the same way that they would use dialogues, readings, etc. for this purpose. In this way, they will not only diversify their teaching style, but also inject a recreational element into the instructional component of the language course. On the other hand, teachers may wish to utilize form-based puzzles as complementary exercise material. Contentbased puzzles are more useful for the building up of communicative competence. Since the content of these puzzles must be deciphered, assessed and interpreted in the target language they are effective devices for cultivating comprehension. And since they require the formulation of a solution strategy in the target language, they are also useful for the teaching/learning of speech production. If the solutions are discussed orally in class, they can also be used to stimulate verbal interaction. Teachers may elicit solutions with prepared questions, or they may allow students to discuss their solutions by themselves. Every content-based puzzle 
is, of course, a source of grammatical and lexical information, and they may be used, additionally, to highlight some point of structure or vocabulary. These puzzles can also be used as composition stimuli; i.e., teachers can ask their students to write their own puzzles and these can be solved by everyone in the class.

These are just some suggestions on the pedagogical uses of recreational problem solving activities in language courses. ${ }^{17}$ Skilled teachers will, needless to say, discover other uses for these puzzles. The central idea behind their incorporation into language programs is that they are effective teaching/learning devices. They diversify teaching techniques and inject an important recreational element into the learning process.

\section{University of Toronto}

\section{NOTES}

1 The following are just a few of the more recently published textbooks and readers for Italian which contain recreational activities: Janice M. Kozma, Carosello (New York: Holt, Rinehart and Winston, 1978); Ferdinando Merlonghi, Franca Merlonghi and Joseph A. Tursi, Oggi in Italia (Boston: Houghton Mifflin, 1978); Anthony Mollica and Angela Convertini, L'Italia racconta (Toronto: Copp Clark, 1979); Graziana Lazzarino et Al., Prego (New York: Random House, 1980).

2 See, for a good summary of the literature, J.M. Stephens and E.D. Evans, Development and Classroom Learning (New York: Holt, Rinehart and Winston, 1973), pp. 251-88.

3 Henry E. Dudeney, The Canterbury Puzzles (New York: Dover, 1958), p. 13.

4 Anthony Mollica, "Visual Puzzles in the Second-Language Classroom," Canadian Modern Language Review, 37 (1981), 620.

5 See, for example, W.R. Lee, Language Teaching Games and Contests (Oxford: Oxford University Press, 1965); Alice Omaggio, Games and Simulations in the Foreign Language Classroom (Arlington, Virginia: Center for Applied Linguistics, 1979); Anthony Mollica, "Games and Language Activities in the Italian High School Language Classroom," Foreign Language Annals, 12 (1979), 347-54; and Mollica, "Visual Puzzles," op. cit.

6 H.H. Clark, "Linguistic Processes in Deductive Reasoning," in Thinking: Readings in Cognitive Science, ed. P.N. Johnson-Laird and P.C. Wason (Cambridge: Cambridge University Press, 1977), p. 98.

7 S. Scribner, "Modes of Thinking and Ways of Speaking: Culture and Logic Reconsidered," in Thinking: Readings in Cognitive Science, cit., p. 497.

8 Martin Gardner, The Sixth Book of Mathematical Games from Scientific American (New York: Scribner's, 1971), ix.

9 Mollica, "Visual Puzzles," op. cit.

10 See also Dino Bressan, "Crossword Puzzles in Modern Language Teaching," Audio-Visual Language Journal, 8 (1970), 93-95.

11 The term "alphametic" was coined by J.A.H. Hunter, Brain Teasers (New York: Bantam, 1965), p. 119.

12 See Mollica, "Visual Puzzles," op. cit.

13 A good source of form-based puzzles (in English) is A. Ross Eckler, Word Recreations (New York: Dover, 1979). This is a useful book because it describes various puzzle types. 
14 I proposed these puzzle types in two previous articles: "Puzzles in Language Teaching," Canadian Modern Language Review, 35 (1979), 269-77, and "Mathematical Games in Foreign Language Courses," Rassegna Italiana di Linguistica Applicata, 12 (1980), 195-204.

15 For a good selection of logical deductions see, C.R. Wylie, 101 Puzzles in Thought and Logic (New York: Dover, 1957).

16 One good collection of both form-based and content-based puzzles is J.P. Adams, We Dare You to Solve This! (New York: Berkley, 1957).

17 For other suggestions, see Mollica, "Visual Puzzles," op. cit. 